\title{
Association of ApoE isoforms with COVID-19 outcomes: a world-wide epidemiological study
}

\author{
Gunanidhi Dhangadamajhi $^{1}\left[\right.$ [D $\cdot$ Swayamparna Mishra ${ }^{1} \cdot$ Payal Mukherjee $^{1}$
}

Received: 28 June 2021 / Accepted: 13 August 2021 / Published online: 18 August 2021

(c) Japan Human Cell Society 2021

To the Editor,

Recent studies in UK Cohort [1,2] documenting increased COVID-19 incidence and deaths in populations homozygous for apolipoprotein $\mathrm{E}$ (ApoE) $\varepsilon_{4 / /} \varepsilon_{4}$ isoforms compared to $\varepsilon_{3 /} \varepsilon_{3}$ with heightened significance is apprehensive. Furthermore, increased susceptibility of $\varepsilon_{4 /} \varepsilon_{4}$ brain cells to SARS-CoV-2 infection in vitro [3] define its possible causal association for COVID-19 outcomes including neurological defects. However, distribution of ApoE isoforms vary amongst populations and their definitive robust association beyond UK cohort is still unknown. This tempted us to conduct a world-wide genetic association study of ApoE isoforms with COVID-19 outcomes.

Literature search on PubMed and Google scholars was conducted to retrieve ApoE isoforms data from healthy control across different countries of global population from relevant published papers. Papers with insufficient data and/ or country name were excluded. Available data were subjected to Hardy Weinberg Equilibrium (HWE). Data showing deviation were excluded from analysis. Data pooling was carried out when more than one data sets were available for a particular country. Country wise frequency of all three ApoE isoforms $\left(\varepsilon_{2}, \varepsilon_{3}\right.$ and $\left.\varepsilon_{4}\right)$ were determined. COVID-19 data (incidence and deaths per millions of population) accessed on 16th January, 2021 from worldometer site (https://www. worldometers.info/coronavirus/) were associated with frequency distribution of different ApoE isoforms by Spearman's correlation coefficient analysis in GraphPad Prism (version 5.0). Data of 16th January, 2021 (latest available before massive vaccination drive) was used to nullify the possible confounding effect due to vaccination disparity

Gunanidhi Dhangadamajhi

gunarmrc@gmail.com

1 Department of Biotechnology, Maharaja Sriram Chandra Bhanja Deo University (Erstwhile North Orissa University), Mayurbhanj, Baripada, Odisha 757003, India across the countries. A $p$ value $<0.05$ was considered significant.

ApoE data of 103,414 healthy controls from 228 studies belonging to 66 countries were finally enrolled which showed varied ranges of distribution for $\varepsilon_{2}(0.625-19.1 \%)$, $\varepsilon_{3}(52.09-92.57 \%)$ and $\varepsilon_{4}(4.45-28.59 \%)$ (S1). Correlation analysis revealed significant but weak inverse relationship of $\varepsilon_{4}$ allele with COVID-19 susceptibility ( $p=0.033$, Spearman's $r=-0.263$ ). Correlation analysis of ApoE alleles with COVID-19 morbidity and mortality was conducted separately for populations of different genetic ancestry (such as Asia, Africa, America and Europe). Consistently, $\varepsilon_{4}$ allele was negatively correlated with COVID-19 outcomes (for incidence: $p=0.0181, r=-0.6$; for deaths: $p=0.0078$ and $r=-0.6571)$ in Asia, only. Interestingly, a significant positive correlation was observed between $\varepsilon_{3}$ allele frequency and COVID-19 outcomes (for incidence: $p=0.0045$ and $r=0.689$; for deaths: $p=0.0011$ and $r=0.757$ ) in Asia, whereas in Europe, this association reached to significant level with mortality ( $p=0.048, r=0.383$ ) only. None of the alleles were found to be correlated with COVID-19 outcomes in Africa and America (Table 1).

Contrasting to UK cohort $[1,2]$, we did not observe $\varepsilon_{4}$ isoform as the risk factor of COVID-19 even in Europeans. Since, UK cohort were older age population and had comorbidities, they were more vulnerable to COVID-19. Furthermore, complete exclusion of individuals having one or more of the comorbidities being not done, COVID-19 risk in $\varepsilon_{4 /} \varepsilon_{4}$ should not be proclaimed as independent to pre-existing ApoE e4 associated comorbidities. Our study which includes apparently healthy control from across the world of all age groups negates the impact of comorbidity, though age groups stratified association of ApoE isoforms with COVID-19 could not be performed as age distribution of genotypes data were not available. Moreover, our findings of inconsistency in associations of ApoE variants with COVID-19 outcomes should not be surprised, since the distribution of ApoE isoforms widely vary across populations 
Table 1 Association of ApoE isoforms with COVID-19 outcomes

\begin{tabular}{|c|c|c|c|c|c|c|}
\hline \multirow[t]{2}{*}{ ApoE isoforms/ populations } & \multicolumn{2}{|l|}{$\varepsilon_{2}$} & \multicolumn{2}{|l|}{$\varepsilon_{3}$} & \multicolumn{2}{|l|}{$\varepsilon_{4}$} \\
\hline & $\mathrm{C} / \mathrm{M}$ & $\mathrm{D} / \mathrm{M}$ & $\mathrm{C} / \mathrm{M}$ & $\mathrm{D} / \mathrm{M}$ & $\mathrm{C} / \mathrm{M}$ & $\mathrm{D} / \mathrm{M}$ \\
\hline Asia $(N=15)$ & 0.458 & 0.226 & $\begin{array}{c}0.0045 \\
(0.689)\end{array}$ & $\begin{array}{l}0.0011 \\
\quad(0.757)\end{array}$ & $\begin{array}{l}0.0181 \\
(-0.6)\end{array}$ & $\begin{array}{l}0.0078 \\
\quad(-0.6571)\end{array}$ \\
\hline Africa $(N=10)$ & 0.260 & 0.293 & 0.803 & 0.803 & 0.777 & 0.726 \\
\hline America $(N=13)$ & 0.437 & 0.312 & 0.816 & 0.958 & 0.654 & 0.689 \\
\hline Europe $(N=27)$ & 0.946 & 0.765 & 0.112 & $\begin{array}{l}0.048 \\
\quad(0.383)\end{array}$ & 0.434 & 0.338 \\
\hline Total $(N=66)^{\mathrm{a}}$ & 0.458 & 0.913 & 0.129 & 0.104 & $\begin{array}{l}0.033 \\
\quad(-0.263)\end{array}$ & 0.107 \\
\hline
\end{tabular}

Values inside the box are p value. Spearman's correlation coefficient values ( + for positive correlation and - for negative correlation) are given in parentheses for which a significant $p$ value of $<0.05$ is observed $C / M$ cases/millions, $D / M$ deaths/millions, $N$ number of studies

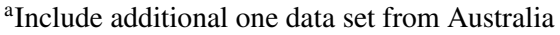

and that functionality of these isoforms are shown to be dependent on their race/ethnicity specific linkage disequilibrium (LD) and complex interactions with other genetic variants in ApoE clusters [4]. The underlying differences in association of ApoE isoforms in the present study can be explained by similar population specific heterogenous effects of ApoE isoforms. Therefore, definitive association of ApoE isoforms towards COVID-19 outcomes is challenging and seems to be more complex. The fact that angiotensin-converting enzyme 2 (ACE2) (an important host receptor for SARS-Cov2 infection and one of the several determinants for population variability of COVID-19 outcomes) and ApoE co-express in lungs alveolar cells [5], the main target for SARS-Cov2 infection. Interestingly, presence or absence of ACE2 in ApoE knockout mice is described to modulate ApoE related altered physiology [3, 6, 7]. However, such connection between ACE2 and ApoE are yet to be established in COVID-19. We hypothesize that population specific variable expression of ACE2 and other relevant genes in ApoE clusters may affect association of ApoE isoforms towards COVID19 outcomes. Therefore, well designed observational studies across various populations of different genetic backgrounds are sought to establish a definitive role of ApoE in COVID19.

Supplementary Information The online version contains supplementary material available at https://doi.org/10.1007/s13577-021-00596-y.

Acknowledgements We acknowledge our Vice Chancellor, Prof K. $\mathrm{K}$ Basa for his encouragement and support from DST-FIST of our Department at Maharaja Sriram Chandra Bhanja Deo University. We also thankfully acknowledge the authors whose contribution have been cited.

Author contributions Dr. G Dhangadamajhi: conceived the original idea, collected and analyzed the data, wrote the final draft of manuscript; Swayamparna Mishra and Payal Mukherjee: collected data and performed preliminary data analysis, prepared the pre-draft.
Funding We did not receive a fund of any kind concerning to this work.

\section{Declarations}

Conflict of interest All authors declare no conflict of interest.

\section{References}

1. Kuo C-L, Pilling LC, Atkins JL, Masoli JA, Delgado J, Kuchel GA, et al. ApoE e4e4 genotype and mortality with COVID-19 in UK Biobank. J Gerontol Ser A. 2020;75(9):1801-3.

2. Kuo C-L, Pilling LC, Atkins JL, Masoli JA, Delgado J, Kuchel GA, et al. APOE e4 genotype predicts severe COVID19 in the UK Biobank community cohort. J Gerontol Ser A. 2020;75(11):2231-2.

3. Wang C, Zhang M, Garcia G Jr, Tian E, Cui Q, Chen X, et al. ApoE-isoform-dependent SARS-CoV-2 neurotropism and cellular response. Cell Stem Cell. 2021;28(2):331-42.

4. Kulminski AM, Shu L, Loika Y, Nazarian A, Arbeev K, Ukraintseva S, et al. APOE region molecular signatures of Alzheimer's disease across races/ethnicities. Neurobiol Aging. 2020;87:141-e1.

5. Gkouskou K, Vasilogiannakopoulou T, Andreakos E, Davanos N, Gazouli M, Sanoudou D, et al. COVID-19 enters the expanding network of apolipoprotein E4-related pathologies. Redox Biol. 2021. https://doi.org/10.1016/j.redox.2021.101938.

6. Chen L-J, Xu Y-L, Song B, Yu H-M, Oudit GY, Xu R, et al. Angiotensin-converting enzyme 2 ameliorates renal fibrosis by blocking the activation of mTOR/ERK signaling in apolipoprotein E-deficient mice. Peptides. 2016;79:49-57.

7. Jin H-Y, Chen L-J, Zhang Z-Z, Xu Y-L, Song B, Xu R, et al. Deletion of angiotensin-converting enzyme 2 exacerbates renal inflammation and injury in apolipoprotein E-deficient mice through modulation of the nephrin and TNF-alpha-TNFRSF1A signaling. J Transl Med. 2015;13(1):1-16.

Publisher's Note Springer Nature remains neutral with regard to jurisdictional claims in published maps and institutional affiliations. 